\title{
Fuzzy Modelling of Air Preparation Stage in an Industrial Exhaust Air Treatment Process
}

\author{
Aleš Šink ${ }^{1}$ Gašper Mušič ${ }^{2}$ \\ ${ }^{1}$ Inea d.o.o., Slovenia, ales.sink@inea.si \\ ${ }^{2}$ Faculty of Electrical Engineering, University of Ljubljana, Slovenia, gasper.music@fe.uni-lj.si
}

\begin{abstract}
The paper is focused on practical aspects of advanced nonlinear identification method applied to a real industrial process. Fuzzy identification is used to model the air preparation stage within a system for reducing nitrogen oxides (NOx) emissions in exhaust air from the dryers and ovens in a factory of automotive catalytic converters. The system for NOx emissions reduction operates efficiently in predetermined temperature and air flow ranges of the exhaust air only. Due to those conditions, exhaust air from the dryers and ovens must be prepared in advance by controlling the ventilator speed and fresh air and exhaust air dampers positions. At the same time operating conditions of dryers and ovens have to be maintained within defined ranges. Currently used control system of the exhaust air preparation shows some deficiencies, so a feasibility study of possible improvements has been carried out. Modelling presented in this paper has been used to evaluate and compare control solutions. The results show such an improvement is feasible. The proposed control system can be ready for implementation in the real process with minor changes of the controller parameters and supervisory logic settings.
\end{abstract}

Keywords: fuzzy logic, Takagi-Sugeno model, catalytic converter, emission reduction, process control

\section{Introduction}

Emissions of pollutants are a challenging problem in many contemporary industrial processes. In particular, emissions of atmospheric pollutants in exhaust air have direct influence on quality of the living conditions in the neigbouring areas of industrial plants, as well as other important environmental impacts, e.g. climate change.

The nitrogen oxides (NOx) emissions in the exhaust air can be effectively reduced by catalytic converters, which are based on the same operation principles as used in internal combustion engine exhaust system in traffic vehicles. Industrial catalytic converters typically work on selective catalytic reduction (SCR) principle, although also selective non-catalytic reduction (SNCR) based systems have been used, e.g. in waste incineration plants. With SCR, a gaseous reductant is added to the stream of exhaust air, typically anhydrous ammonia, aqueous ammonia or urea, which reacts with gas mixture in the exhaust air and the catalyst to form molecular nitrogen, water and carbon dioxide.

To achieve the (near) optimal operation of the catalytic converter unit, the incoming production process exhaust air must be properly conditioned. This involves control of flow, pressure and the temperature.

The currently used control system of the exhaust air preparation stage in the process under investigation shows some deficiencies, so a feasibility study of possible improvements has been carried out. A substantial part of the feasibility study was development of a mathematical model, which is presented in this paper. The model was necessary in order to be able to experiment with control system. The presentation focuses on practical aspects of deriving a model by fuzzy identification based on actual production data.

\section{Air preparation process}

The system considered in the paper is a part of the catalytic converters production plant. The produced catalytic converters are used to reduce NOx emissions from internal combustion engine driven vehicles.

Due to the used production technology, the NOx emissions are present in the production process itself so an emissions reduction unit is installed. The operating conditions are varying substantially with the changing production. Therefore an air preparation stage is installed in between the production process and the catalytic converter (Fig. 1). This way a stable operating regime of the NOx emissions reduction unit is achieved.

The process contains a pipe system delivering exhaust air from ovens and dryers, equipped with controllable dampers and ventilators; a set of pressure/differential pressure and temperature sensors, and damper position sensors is installed. Low pressure is maintained to transport the air out of the process and the air flow is calculated from differential pressure reading within the catalyst converter unit, taking into account air pressure and air temperature. According to specifications the control relevant signals are: air flow, pressure before ventilator, differential pressure over the ventilator, ovens exhaust pressure and dryers exhaust pressure.

The currently operating system involves automatic control of the exhaust air preparation system, but the control system is not operating in a closed-loop manner. The con- 


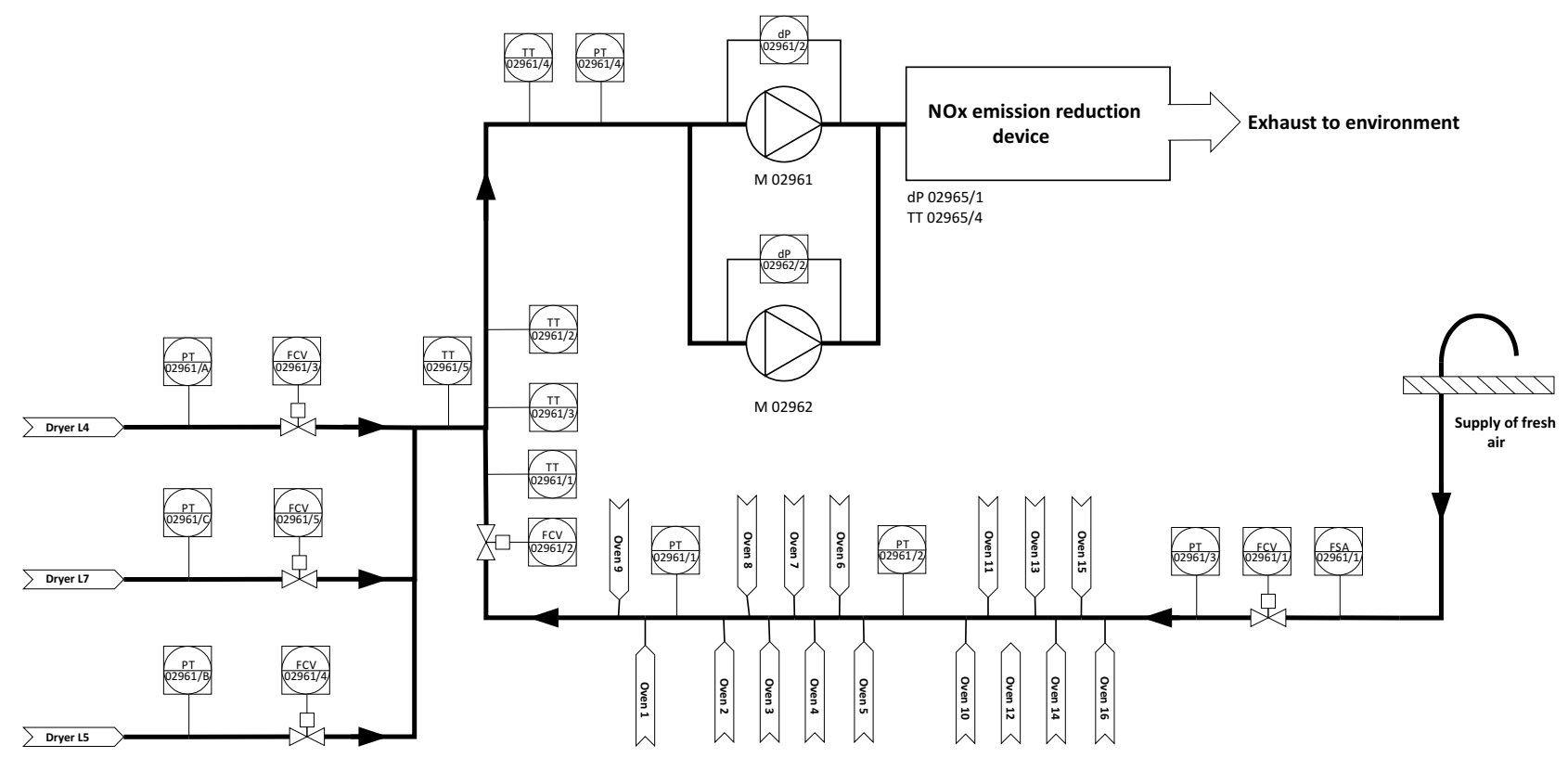

Figure 1. Air preparation process.

trol logic is programmed in accordance with a set of predefined rules based on operator expertise. The manipulated signals of dampers and ventilators are changed stepwise, with large intermediate intervals to allow the process to settle in the changed operating point. This induces substantial delays before the process adapts to any changes in the exhaust air conditions. Therefore a feasibility study was carried out to show potential improvements in reduction of emissions employing closed-loop control in the air preparation stage.

The study is based on simulation so a mathematical process model was needed. Experimentation on the actual system was not possible due to potential environmental hazard. In fact, the system involves several safety measures to prevent this and experimentation with the air preparations stage potentially induces the production shutdown, which is not acceptable.

\section{Fuzzy identification}

Due to nonlinear nature of the process dynamics, fuzzy logic (Zadeh, 1965) was chosen as a modelling framework and Takagi-Sugeno (TS) type model was developed. The model parameters were identified by actual production data, a separate dataset was used for model validation. The intended use of the model is to simulate a closed loop control system with some typical operation scenarios.

\subsection{Takagi-Sugeno fuzzy model}

With TS fuzzy model an arbitrary nonlinear system can be approximated by smooth interpolation of affine local models (Takagi and Sugeno, 1985). Every local model contributes to global model in the frame of fuzzy clusters in the space described by membership functions.

If the input data is denoted as $X=\left[x_{1}, x_{2}, \ldots, x_{n}\right]^{T}$ and the output data as $Y=\left[y_{1}, y_{2}, \ldots, y_{n}\right]^{T}$, then the model in
TS form (Takagi and Sugeno, 1985) is written as a set of rules:

$$
R_{i}: \text { if } x_{k} \text { is } A_{i} \text { then } \hat{y}_{k}=\phi_{i}\left(x_{k}\right) \quad i=1, \ldots, c
$$

Vector $x_{k}$ represents the input data in premise while $\hat{y}_{k}$ is the output of the fuzzy model at time instant $k$. Premise vector $x_{k}$ relates to fuzzy sets $\left(A_{1}, \ldots, A_{c}\right)$, where every fuzzy set $A_{i}(i=1, \ldots, c)$ is characterized by a real valued membership function $\mu_{A_{i}}\left(x_{k}\right)$ or $\mu_{i k}: R \rightarrow[0,1]$ representing the membership degree of $x_{k}$ with respect to fuzzy set $A_{i}$. Functions $\phi_{i}($.$) are in general arbitrary smooth func-$ tions, while mostly linear or affine functions are used.

The model output in (1) can be expressed as:

$$
\hat{y}_{k}=\frac{\sum_{i=1}^{c} \mu_{i k} \phi_{i}\left(x_{k}\right)}{\sum_{i=1}^{c} \mu_{i k}}
$$

Equation (2) can be simplified by introducing $\beta_{i}\left(x_{k}\right)$ defined as:

$$
\beta_{i}\left(x_{k}\right)=\frac{\mu_{i k}}{\sum_{i=1}^{c} \mu_{i k}}, i=1, \ldots, c
$$

In this way the degree of fulfillment of a fuzzy rule is given in a normalized form. $\sum_{i=1}^{c} \beta_{i}\left(x_{k}\right)=1$ independently of $x_{k}$, as long as $\beta_{i}\left(x_{k}\right)$ is not zero (which can be easily assured by extending membership functions over the whole range of $x_{k}$ ).

Joining (2) and (3) results in:

$$
\hat{y}_{k}=\sum_{i=1}^{c} \beta_{i}\left(x_{k}\right) \phi_{i}\left(x_{k}\right), k=1, \ldots, n
$$

The output is often defined as a linear combination of the consequence states:

$$
\phi_{i}\left(x_{k}\right)=x_{k} \theta_{i}, i=1, \ldots, c, \theta_{i}^{T}=\left[\theta_{i 1}, \ldots, \theta_{i(p)}\right]
$$


The vector of fuzzified input variables at time instant $k$ is written as:

$$
\psi_{k}=\left[\beta_{1}\left(x_{k}\right) x_{k}, \ldots, \beta_{c}\left(x_{k}\right) x_{k}\right], k=1, \ldots, n
$$

which implies fuzzified data matrix:

$$
\Psi^{T}=\left[\psi_{1}^{T}, \psi_{2}^{T}, \ldots, \psi_{n}^{T}\right]
$$

If the coefficient matrix for the overall set of rules is written as $\Theta^{T}=\left[\theta_{1}^{T}, \ldots, \theta_{c}^{T}\right]$, (4) can be modified to:

$$
\hat{y}_{k}=\psi_{k} \Theta
$$

and in compact form, which describes the overall data set relations:

$$
\hat{Y}=\Psi \Theta
$$

where $\hat{Y}$ is a vector of model outputs $\hat{y}_{k}$ and $k=1, \ldots, n$ $\left(\hat{Y}=\left[\hat{y}_{1}, \hat{y}_{2}, \ldots, \hat{y}_{n}\right]^{T}\right)$.

The fuzzy model (7) is often denoted as affine TakagiSugeno model and can be used to approximate any real valued continuous function with arbitrary precision (Kosko, 1994; Wang and Mendel, 1992; Ying and Chen, 1997). This can be proved by Stone-Weierstrass theorem. Any real valued continuous function can be approximated with a fuzzy model (Lin, 1997).

\subsection{Fuzzy clustering}

The TS model can be derived from available process data by identifying the structure and the parameters of the local models (Takagi and Sugeno, 1985). Structure identification includes an estimation of the cluster centers (antecedent parameters), which is usually done by fuzzy clustering. Then for each cluster the sub-model's parameters are estimated, which is usually done with a least-squares method (Chiu, 1994). Clustering can be performed in the input space only or in the product space of the input and output. The later is more general and is commonly termed shortly as clustering in the product space

The basic principle of fuzzy clustering is depicted in Fig. 2(a). Here the data is clustered into two groups with prototypes $v_{1}$ and $v_{2}$, using the Euclidean distance measure (Babuška, 1998).

If-then rules of the fuzzy model are extracted by clusters projection onto the axes. The clusters can be ellipsoids with adaptively determined shape (Gustafson and Kessel,

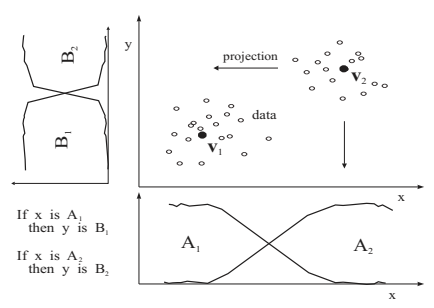

(a) Rule-based interpretation of fuzzy clusters.

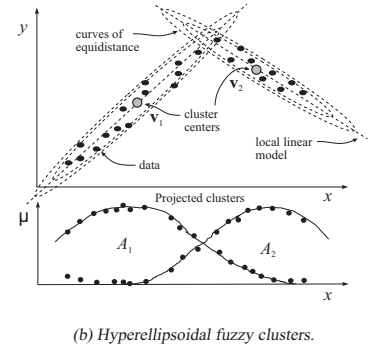

(b) Hyperellipsoidal fuzzy clusters.
Figure 2. Identification by fuzzy clustering (Babuška, 1998).

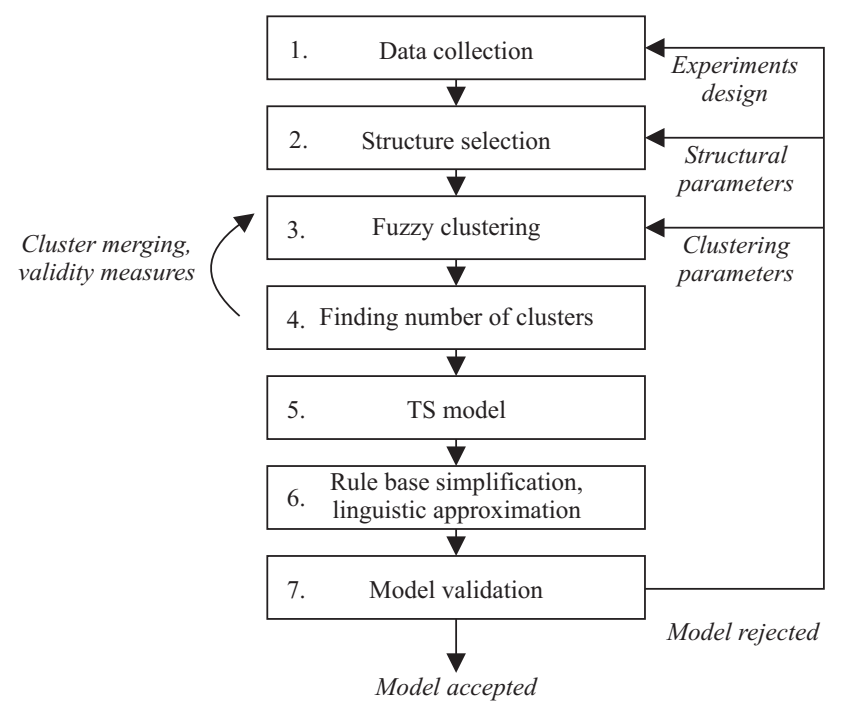

Figure 3. Identification approach based on fuzzy clustering (Babuška, 1998).

1979), see Fig. 2(b). From such clusters, the antecedent membership functions and the consequent parameters of the TS model can be identified (Babuška, 1998). Each obtained cluster is represented by a rule in the TS model. The principle of system identification employing fuzzy clustering consists of a series of steps depicted in Fig. 3.

The procedure is supported by dedicated software tools, such as Fuzzy Modelling and Identification Toolbox for Matlab (FMID), which was used in the presented work. Implementation of some of the steps of Fig. 3 will be discussed in the following sections.

\section{Data collection}

Data collection is a key step that largely determines the quality of the modelling result. Proper identification experiments have to be designed and this is a very problematic step in several industrial processes, in particular when a process is already in operation. In some cases the process shutdown is impossible for the reason of cost or safety; in others the standalone experimentation is not possible. Because of process interconnections, it is often required that several process stages operate in line with the process under observation.

When experimentation is performed during operation, typically a number of operating restrictions have to be observed, which severely limits the experimentation possibilities.

In the presented case the air preparation process cannot run independently of main production process which delivers the polluted exhaust air. At the same time nominal operating regime has to be maintained within the main process to avoid product scrap or even automatic process shutdown, and furthermore, the emissions should stay within allowed ranges.

The first identification attempt was done with signals acquired during the control commissioning. Signals ac- 
quired while the operation of control logic was tested were used as input-output data. The modelling results were not useful, because it turned out that the testing has not covered adequately large portion of the input-output space. Only manipulated values could be directly changed during testing while several other signals depend on process operating conditions that were not directly controlled. Large number of situations was simply not covered by the testing procedures, which is quite common in complex processes.

The second attempt was based on data acquired during a longer process operation testing period. The problem in this case was the built-in data compression within the data acquisition system, which writes the data only on sufficient changes. The problem was solved by interpolating available sensor measurements and by stepwise holding the manipulated values. The results were resampled by $1 s$ interval.

\section{Structure selection}

According to (Babuška, 1998) the structure selection in fuzzy modelling involves a set of tasks, such as: a choice of input and output variables, representation of the systems' dynamics, and a choice of the fuzzy models granularity.

\subsection{Input and output variables}

The control relevant output signals were specified by the customer, the same were chosen as model outputs. The inputs were chosen in accordance with the known physical background of the process. Also an expertise of the process operators was considered.

Additionally, the possibilities of data analysis with regard to determination of influential variables were tested (Glavan et al., 2013). The analysis has not brought any new insights into the input output dependencies. This indicates that for well-known processes data analysis methods are not superior to human expertise. Inputs of some of the submodels depend on the outputs of other submodels. Fig. 4 shows the initial structure of the overall process model.

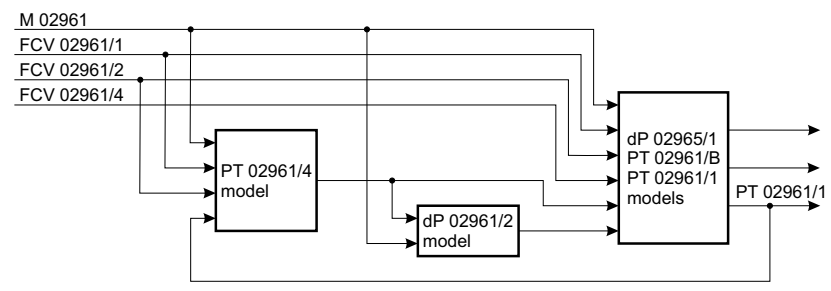

Figure 4. Initial model structure.

While individual models show good matching with the process data, the overall model of Fig. 4 exhibits large modelling error. The reason is in the error accumulation in PT 02961/1, PT 02961/4 and dP 02961/2 models (signals are labelled in accordance with process scheme in Fig. 1). Therefore, another model structure was chosen, which consists of separate models for all quantities of interest, which directly build on measured data. E.g., the model for air pressure in the ovens outlet is shown in Fig. 5.

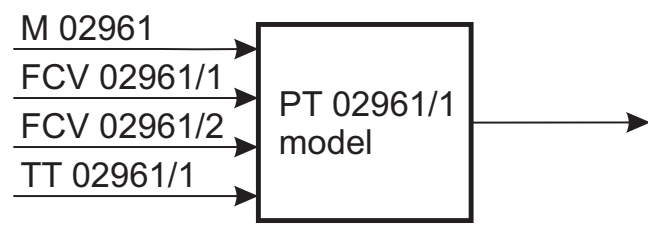

Figure 5. Fuzzy model of air pressure in the ovens outlet.

\subsection{Representation of the systems' dynamics}

The main design issue here was to choose the number of delayed input and output samples used as model regressors. The choice was determined by an iterative procedure, during which we started the identification with one sample delay on input and output, and then repeated the identification with increasing number of delayed outputs up to four. The effect of adding additional delayed input was also tested as well as changes of sampling interval to 2 and $3 s$.

The quality of derived models was compared by variance accounted for (VAF) performance index (Babuška, 1998)

$$
V A F=100 \%\left[1-\frac{\operatorname{var}\left(y_{1}-y_{2}\right)}{\operatorname{var}\left(y_{1}\right)}\right]
$$

Among others, the above procedure led us to adjust the sampling period of the acquired data; for the further experimentation a $3 s$ sampling interval was chosen. This allowed us to use a low number of delayed signals as model regressors (2 to 3 ), which prevents overfitting and improves model generalization capability.

\subsection{Fuzzy models granularity}

When fuzzy clustering is applied to generate fuzzy models from data, the main parameter that must be chosen with regard to granularity is the number of clusters. The applied strategy here was to start with a moderate number of clusters (e.g. 5) and then experiment in a similar manner as above, gradually increasing the number of clusters up to 8 , and taking VAF index as performance measure.

\section{Fuzzy clustering and model valida- tion}

All models for the control relevant variables defined in Sect. II were built in a similar manner by using FMID tool and experimenting with main parameters.

Fig. 6 shows the performance of the best model for air pressure in the ovens outlet with respect to the identification signal and the validation signal.

The VAF value is $67.4 \%$ for the identification signal and $28.9 \%$ for the validation signal. The result of validation is rather low but the reason is in the nature of the process operation. The observed pressure largely depends 

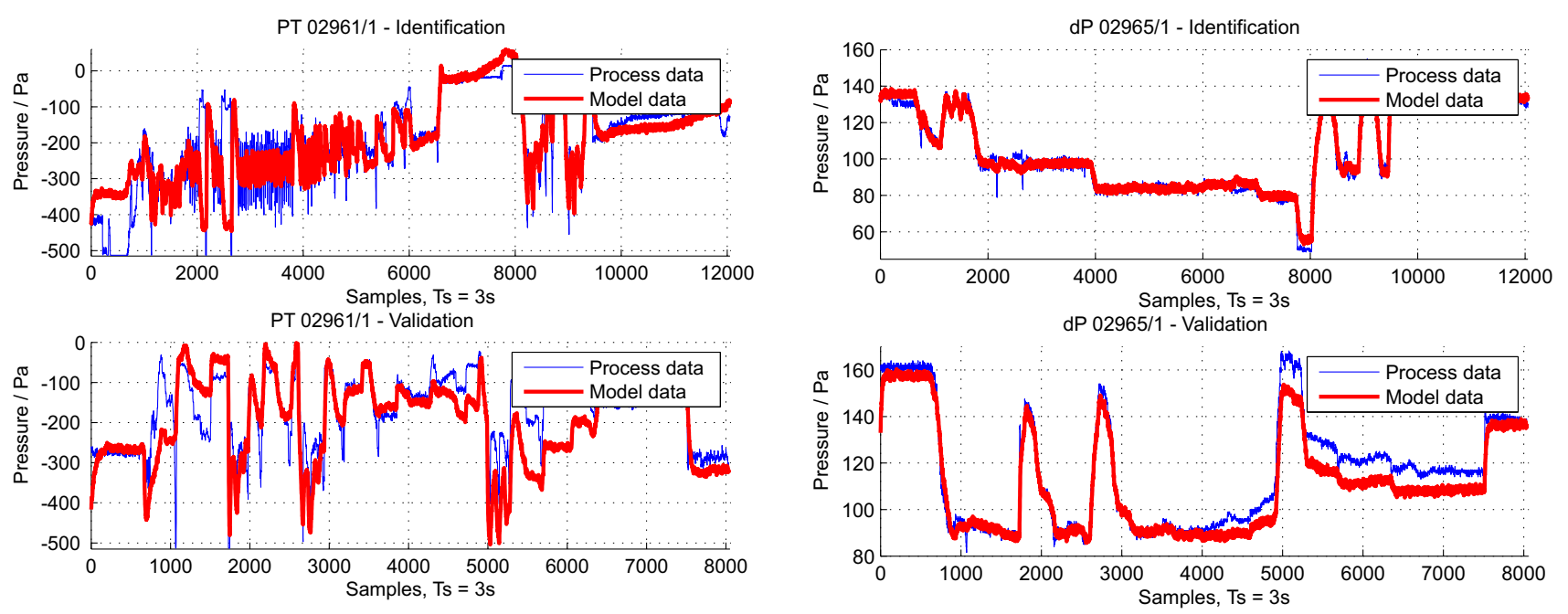

Figure 6. Performance of the fuzzy model of air pressure in the ovens outlet.

on the number of the operating ovens. This parameter was not available in the process database so it could not be included as an input. It is assumed that occasional large deviations from the measured validation signals are due to change of this condition.

Fig. 7 shows a similar graph for the model of differential pressure, which is the basis for the air-flow calculation.

The VAF value is $97.8 \%$ for the identification signal and $95.0 \%$ for the validation signal, which is much better than in the previous case. This indicates that chosen modelling method can be very effective when proper inputoutput data is provided.

\subsection{Comments on resulting model perfor- mance}

We estimate that derived fuzzy models are of sufficient quality to test and compare various control solutions. There are noticeable deviations in responses of the PT 02961/1 model and the real system, but these are presumably mainly caused by varying conditions in the quantity of supplied exhaust air due to variable number of operating stages in the main manufacturing process.

The most relevant control variable is the air flow FIA $02965 / 2\left(\Phi_{\text {air }}\right)$ through the NOx emission reduction device, which is calculated based on dP 02965/1 ( $\Delta p[\mathrm{~Pa}])$ and TT 02965/4 $\left(T\left[{ }^{\circ} C\right]\right)$ readings as follows:

$$
\begin{gathered}
\Phi_{\text {air }}=3600 \cdot A \cdot v_{\text {air }} \quad\left[\mathrm{m}^{3} / \mathrm{h}\right] \\
v_{\text {air }}=\sqrt{\frac{2 \Delta p}{R \cdot \rho}} \quad[\mathrm{m} / \mathrm{s}] \\
\rho=1.293 \frac{273}{T+273} \quad\left[\mathrm{~kg} / \mathrm{m}^{3}\right]
\end{gathered}
$$

where $R=4.6285$ and $A=2.1316 \mathrm{~m}^{2}$.

As presented above, the $V A F$ index obtained during validation shows good quality of dP 02965/1 model, which

Figure 7. Performance of the fuzzy model of differential pressure in the catalyst converter unit.

indicates the obtained model is of sufficient quality for testing air flow control strategies.

By stepwise changes of the manipulated variables the model behavior was also qualitatively evaluated, and all the signals change in accordance with experience on the real process.

Additional temperature measurements were considered as model inputs during the identification. When testing control strategy these signals are not available, therefore a set of disturbance generating models were additionally identified. Major disturbances were generated, in particular the air temperatures in different parts of the system.

These models are not very precise but enable generation of disturbances during simulated experiments with control system. Control robustness is tested this way.

\section{Control experiments}

As the signals observed in the real process are noisy and disturbed by additional influential variables, these aspects are added to the model used for control experiments. Noise amplitude and frequency bandwidth of the noise filter were chosen in accordance to observed process signals in the manual mode.

\subsection{PID control}

After model was validated and brought close to the actual process behavior by addition of noise, it was used to test various control strategies. As the focus here in this paper is not on control design, only a sample simulated scenario of the air flow is shown in Fig. 8. Note that simple PI controller was used that was tuned in a chosen operating point without noise in the model. After tuning, the control robustness was checked in the presence of noise and disturbances. Among others, additional noise cancelling measures were implemented, such as deadband on manipulated value and error filtering. 

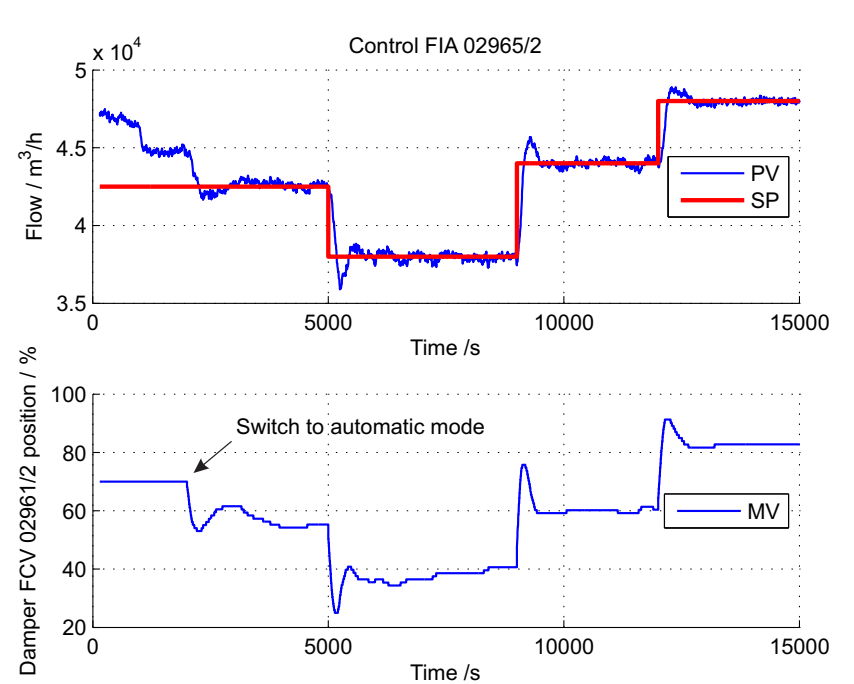

Figure 8. Illustration of the control system performance - air flow.

\subsection{Supervisory logic}

The advantage of the conventional (currently used) openloop control system is the simple accommodation for the process exceptions. These are treated by addition of related rules in the control logic.

The main process exceptions are related to:

- start-up of the air preparation system

- exceptional process values

- invalid pressure set points.

In the following we briefly illustrate how exceptional process values are treated. The observed values are:

- high temperature in the ovens output airflow

- high temperature before the main ventilator

- high emissions concentration on the input

- high differential pressure on the main ventilator.

The desired action in all these exception is to increase the air flow through the system. In the conventional logic this is achieved by simply opening the corresponding damper for a predefined proportion.

In the proposed new control system this is accommodated by additional supervisory logic, which increases setpoint value for airflow at the predefined warning levels. With the low warning level the setpoint is increased for $2000 \mathrm{~m}^{3} / \mathrm{h}$, at the high warning level the setpoint is increased for $5000 \mathrm{~m}^{3} / \mathrm{h}$.

When lower alarm level is reached the system switches to manual mode with fully open dampers and predefined ventilator speed. At higher alarm level the system is shut down with dampers fully open.

The sample simulated operation scenario is shown in Fig. 9, where setpoint changes and related changes in the

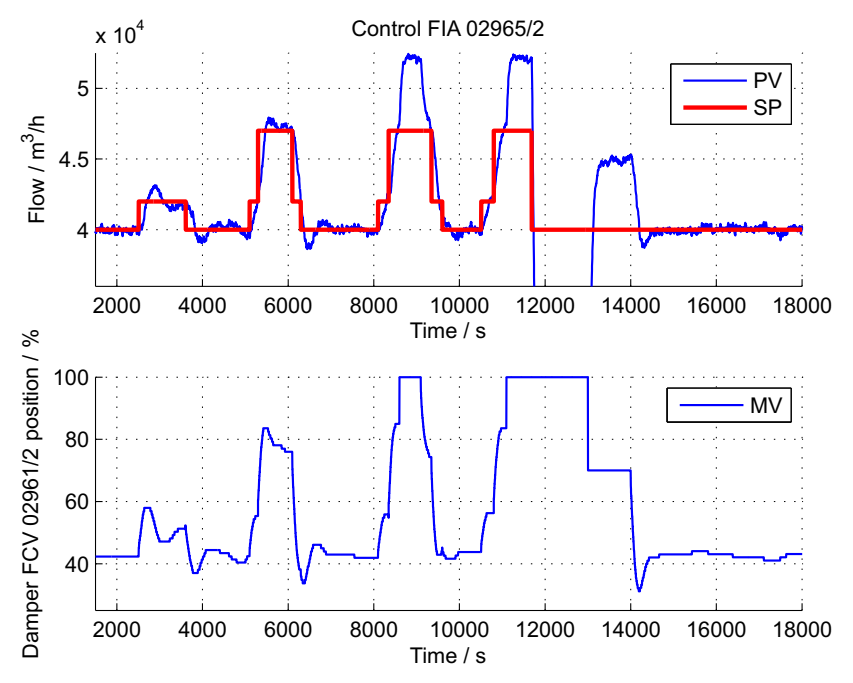

Figure 9. Illustration of the control system performance in the presence of process exceptions.

damper position can be observed, and normal system operation reestablishment can be seen when operation conditions are brought back to normal.

The results show that treatment of the exceptional conditions can be accomodated by adjustments of the setpoint values by supervisory logic.

\section{Conclusions}

The feasibility study shows the control of the air preparation stage could be improved with a moderate investment in the control equipment and related application software. The currently used control system is implemented in the ControlLogix 5570 family of Programmable Logic Controllers (PLCs) with PID control support within the corresponding PLC programming software. The application software could be adjusted by replacing open-loop control logic with PID controllers. Minor parameter adjustments are foreseen mainly due to different scaling of the control signals compared to simulation study.

The chosen modelling approach showed its value in relatively simple identification of the submodels when the proper model structure was determined. Nevertheless, the determination of the structure was a challenging task that was solved by using a-priori knowledge of the system behaviour in combination with experimental adjustments of the main structural parameters. Inferring this automatically from a given data set remains a difficult task, in particular in real industrial processes where possibilities of the experimentation with the input signals are limited, due to technological and safety restrictions.

The focus of the presented model development was in obtaining a model that is representative enough to enable simulated control experiments. We estimate the goal was achieved and results of the simulation experiments are satisfactory. Addition of data related to operating parameters in the main process, in particular the number of operating ovens, would open a way to improve the model and perform a feasibility study involving more advanced con- 
trol strategies. Nevertheless, the potential implementation of such strategies in the industrial process under study is limited with existing control equipment.

\section{References}

R. Babuška. Fuzzy Modeling for Control. Kluwer Academic Publishers, Boston, USA, 1998.

Stephen L. Chiu. Fuzzy model identification based on cluster estimation. J. Intell. Fuzzy Syst., 2(3):267-278, May 1994. ISSN 1064-1246.

Miha Glavan, Dejan Gradišar, Maja Atanasijević-Kunc, Stanko Strmčnik, and Gašper Mušič. Input variable selection for model-based production control and optimisation. The International Journal of Advanced Manufacturing Technology, 68 (9):2743-2759, 2013. ISSN 1433-3015. doi:10.1007/s00170013-4840-1.

D. E. Gustafson and W. C. Kessel. Fuzzy clustering with a fuzzy covariance matrix. In Proc. 18th IEEE Conf. Decision and Control, pages 761-766, Jan 1979.

Bart Kosko. Fuzzy systems as universal approximators. IEEE Trans. Comput., 43(11):1329-1333, November 1994. ISSN 0018-9340. doi:10.1109/12.324566.

Cheng-Jian Lin. SISO nonlinear system identification using a fuzzy-neural hybrid system. Int. J. Neural Systems, 8(3):325337, 1997. doi:10.1142/S0129065797000331.

T. Takagi and M. Sugeno. Fuzzy identification of systems and its applications to modeling and control. IEEE Trans. Systems, Man, and Cybernetics, SMC-15(1):116-132, Jan 1985. ISSN 0018-9472. doi:10.1109/TSMC.1985.6313399.

L. X. Wang and J. M. Mendel. Fuzzy basis functions, universal approximation, and orthogonal least-squares learning. IEEE Trans. Neural Networks, 3(5):807-814, Sep 1992. ISSN 1045-9227.

Hao Ying and Guanrong Chen. Necessary conditions for some typical fuzzy systems as universal approximators. Automatica, 33(7):1333-1338, 1997. ISSN 0005-1098.

L.A. Zadeh. Fuzzy sets. Information and Control, 8(3):338-353, 1965. ISSN 0019-9958. 